\title{
XU HƯỚNG PHÁT TRIỂN PHƯƠNG PHÁP PPP DỤA TRÊN MẠNG LƯỚI CÁC TRẠM CORS
}

\author{
HÀ MINH HÒA \\ Viện Khoa học Đo đạc và Bản đồ
}

\section{Tóm tắt:}

Sụ phát triển nhanh phuơng pháp PPP trong xủ lý các dĩ liệu GNSS trên cơ sở khai thác hiệu quả các trạm CORS là xu hướng đang được các nước nghiên cưu và phát triển tù̀ năm 2000 dụa trên việc phát triển mạnh mẽ các mạng lưới các trạm CORS và sủ dụng các dịch vu của tổ chức IGS. Phương pháp PPP có ư điểm là cho phép định vị nhanh điểm GNSS trong vòng 30'với độ chính xác ở mức dm đến cm. Đây là xu hướng cần phát triển ở Việt Nam để thúc đẩy công tác nghiên cứu, úng dụng và giảng dậy về công nghệ GNSS ở Việt Nam.

\section{1. Đặt vấn đề}

Việc xử lý các dữ liệu GNSS với các khoảng cách lớn khi sử dụng các dịch vụ của tổ chức IGS (International GNSS Service) trong ITRF (International Terrestrial Reference Frame), chúng ta phải giải quyết nhiều vấn đề khoa học - kỹ thuật như tổ chức các dữ liệu GNSS theo các nhóm đo với việc chọn một vệ tinh là, vệ tinh base, và mỗi khi vệ tinh base lặn phải tiến hành chọn lại nhóm vệ tinh (gap) mới, thường xuyên hiệu chỉnh các số hiệu chỉnh đồng hồ các vệ tinh và ảnh hưởng do biến thiên của tầng điện ly và các hiệu ứng địa động lực khác, cải tính do ảnh hưởng của tầng đối lưu, sự trượt chu kỳ sóng mang và quy nguyên khi giải đa trị .v.v... Đối với độ chính xác cao thời gian thu tín hiệu thường đòi hỏi một vài ca đo $24 \mathrm{~h}$.

Như vậy để đạt được độ chính xác ở mức $\mathrm{dm}$ đến $\mathrm{cm}$ thường yêu cần đo nhiều ngày đêm. Đòi hỏi này làm cho thời gian thu tín hiệu vệ tinh với mục đích nhận được kết quả xử lý GNSS độ chính xác cao mất khá nhiều thời gian.

Mặt khác công nghệ GNSS cũng có những nhược điểm cơ bản như ảnh hưởng của hiện tượng đa đường truyền do các vật cản xung quanh trạm thu tín hiệu vệ tinh và sự biến thiên của tầng điện ly trong quá trình đo đạc GNSS đến các trị đo GNSS. Các ảnh hưởng này không cho phép nhận được lời giải Fixed khi giải đa trị theo các hiệu trị đo kép và sẽ gây ra các sai số hệ thống trong các độ cao trắc địa nhận được từ công nghệ GNSS. Để khắc phục các nhược điểm nêu trên trong quá trình xử lý các dữ liệu GNSS người ta phải sử dụng thêm mô hình ngẫu nhiên (stochastic model) dưới dạng phần không tham số (non - parametric) được xác định dưới dạng các hàm được làm trơn biến thiên chậm theo thời gian và triển khai theo kỹ thuật nửa tham số (semi - parametric and penalised least squares techniques), kỹ thuật mô hình hóa ngẫu nhiên lặp (iteractive stochastic modeling technique) hoặc cách tiếp cận dựa trên sóng nhỏ (wavelet based approach) (Satirapod, C., J. Wang, and C. Rizos, 2003). Các kỹ thuật này thường được sử dụng trong các phần mềm xử lý dữ liệu GNSS hiện đại như Bernese, GAMIT/GLOBK .v.v...

Sự ra đời của tổ chức IGS trực thuộc IERS vào tháng 8 năm 1989 tại Edinburgh (Vương quốc Anh) mở ra một kỷ nguyên mới trong việc ứng dụng công nghệ GNSS (xem chi tiết trong Hà Minh Hòa, Nguyễn Ngọc Lâu, 2013). Mạng lưới các trạm IGS (đến ngày 31/12/2017 đã có 505 trạm IGS bao phủ toàn cầu) kết hợp với các mạng lưới VLBI, SLR, LLR, DORIS được sử dụng để xác định các tham số định vị Trái đất (Earth Orientation Parameters - EOP) như giờ toàn thế giới UT1, các tọa độ của $\mathrm{CEP}$ so với IRP (cực quy chiếu IERS), chương động và tuế sai đảm bảo việc chuyển các tọa độ của các vệ 
tinh từ khung quy chiếu sao quốc tế (ICRF) về khung quy chiếu Trái đất quốc tế ITRF và định vị gốc của ITRF chính xác vào tâm vật chất Trái đất khi tính đến sự dịch chuyển của tâm vật chất Trái đất với tốc độ $1 \mathrm{~mm} / 1$ năm, xác định các tọa độ chính xác của các trạm IGS trong ITRF, xác định lịch vệ tinh chính xác, nghiên cứu chuyển dịch của các mảng kiến tạo .v. v...

Các Trung tâm phân tích IGS hiện nay bao gồm Viện Thiên văn trực thuộc Trường đại học tổng hợp Bern (Thuỵ Sĩ); Trung tâm điều khiển vũ trụ Châu Âu nằm ở CHLB Đức; Trung tâm phân tích FLINN trực thuộc Cơ quan Các nguồn Tài nguyên thiên nhiên của Canada (NRCan); Phòng thí nghiệm phản lực (JPL, NASA, Mỹ); Cơ quan Dịch vụ Trắc địa quốc gia (NGS, Mỹ); Trung tâm SOPAC thuộc Viện Đại dương học của trường Đại học tổng hợp Califonia (San Diego, Mỹ).v.v... Trung tâm SOPAC - một trong 7 trung tâm phân tích của IGS có các chức năng sử dụng phần mềm GAMIT tính toán lịch các vệ tinh GNSS chính xác dựa trên các dữ liệu GNSS thu được từ $0-24 \mathrm{~h}$ từ khoảng 45 trạm IGS và cung cấp cho người sử dụng toàn cầu dưới dạng dữ liệu RINEX, các tọa độ và các tốc độ thay đổi tọa độ của các trạm IGS trong ITRF, tính các tham số định vị Trái đất, tính các độ lệch của chuỗi thời gian.v.v... Đối với nước Mỹ, SOPAC triển khai mạng lưới trắc địa GPS thường trực (PGGA) thuộc mạng lưới GPS tích hợp Nam California và mạng lưới các trạm CORS trên toàn lãnh thổ nước Mỹ.

Bên cạnh chức năng duy tu, bảo trì và hiệu chỉnh khung quy chiếu Trái đất quốc tế ITRF vào các thời điểm chuẩn khi tính đến sự chuyển dịch của các mảng kiến tạo và các hiện tượng địa động lực khác, tổ chức IGS còn cung cấp các sản phẩm dịch vụ công nghệ GNSS như các tọa độ không gian của các trạm IGS với độ chính xác ở mức $\pm 2 \mathrm{~cm}$ và các tốc độ thay đổi của chúng theo thời gian trong ITRF hiện hành, lịch các vệ tinh GNSS chính xác ở các mức $\pm 2,5 \mathrm{~cm}, \pm 5$ $\mathrm{cm}, \pm 10 \mathrm{~cm}$, các số hiệu chỉnh các đồng hồ vệ tinh GNSS, các tham số đặc trưng cho độ trễ tầng đối lưu phương thiên đỉnh (TZPD), các tham số chuyển các tọa độ không gian giữa các ITRF được xác định vào các thời điểm chuẩn khác nhau.

Phòng thí nghiệm phản lực (JPL) xác định nội dung điện tử tổng lượng (TEC) trên $1 \mathrm{~m}^{2}$ đường truyền tín hiệu ở tầng điện ly sau mỗi 5 phút từ khoảng 100 trạm thu GDGPS thời gian thực nhờ phép lọc Kalman trong hệ định vị Mặt trời. Bản đồ TEC toàn cầu được sử dụng trong quá trình xử lý các dữ liệu GNSS và phát triển công nghệ VRS. Ngoài ra JPL còn thành lập lịch DE (Development Ephemeris) cho các hành tinh thuộc hệ Mặt trời, đặc biệt đối với Mặt trăng, Mặt trời để tính đến ảnh hưởng hiện tượng triều Trái đất dưới sức hút của Mặt trăng và Mặt trời đến vị trí các trạm thu các tín hiệu GNSS trong quá trình xử lý các dữ liệu GNSS.

Các sản phẩm dịch vụ do tổ chức IGS cung cấp là cơ sở để phát triển các mạng lưới GNSS độ chính xác cao trên phạm vi lớn đáp ứng các yêu cầu triển khai các nhiệm vụ nghiên cứu các hiện tượng địa động lực diễn ra trên bề mặt Trái đất và phát triển các hệ quy chiếu không gian ở các quốc gia. Đặc biệt, việc phát triển các mạng lưới các trạm CORS có gắn kết với các trạm IGS ở các nước không chỉ hỗ trợ cho tổ chức IGS phát triển mạng lưới các trạm IGS để thực hiện các chức năng của mình, mà còn tạo điều kiện cho các nước thực hiện các nhiệm vụ nêu trên dựa trên ITRF.

Ở Châu Âu tổ chức EUREF cũng triển khai hệ ETRS89 tương tự như ITRF. Vào năm 1987 Tiểu ban 1.3a của Hội Trắc địa quốc tế IAG (sau này được gọi là Tồ chức Khung Trái đất quy chiếu châu Âu - EUREF) đã quyết định phát triển Hệ thống quy chiếu Trái đất Châu Âu ETRS89 dựa trên công nghệ GPS (Ihde J., Bruyninx C., 2008). ETRS89 là hệ tọa độ không gian Trái đất được xây dựng dựa trên ellipsoid GRS80. Vào cuối thập kỷ 80 của thế kỷ XX, ITRF ra đời dưới sự quản lý, điều hành của tổ chức IGS trực thuộc tổ chức Dịch vụ quay Trái đất (IERS). Do đó EUREF quyết định phát triển ETRS dựa trên ITRF. Tuy nhiên do ảnh hưởng của chuyển động kiến tạo, các tọa độ của các 
điểm ở các nước $\mathrm{EU}$ thay đổi chậm với tốc độ $2,5 \mathrm{~cm} / 1$ năm, nên vào năm 1989 tại Hội nghị của EUREF tại Firenze (Italia) đã quyết định các điểm gốc (datum) của ETRS89 được bố trí trên phần ổn định của lãnh thổ $\mathrm{EU}$ vào thời điểm chuẩn 1989.0 dựa trên mạng lưới thường trực GNSS EUREF được xác định trong ITRF. Tổng cộng 38 nước $\mathrm{EU}$ với khoảng gần 100 các hãng, các trường Đại học tổng hợp đã tham gia vào mạng lưới thường trực GNSS EUREF. Mạng lưới này gồm 218 trạm GNSS thường trực, trong đó 80 trạm GPS/GLONASS, 87 trạm IGS.

Việc phối hợp giữa các tổ chức IGS và EUREF thúc đẩy việc ứng dụng công nghệ GNSS không chỉ trong lĩnh vực Trắc địa - Bản đồ, mà còn trong nhiều lĩnh vực khoa học - kỹ thuật, kinh tế - xã hội khác.

Theo tài liệu (GNSS Solutions: Network RTK and Reference Station Configuration), trên trạm CORS luôn có sẵn các dữ liệu:

Các dữ liệu differential GPS (DGPS) để xác định vị trí thời gian thực trên thực địa;

Dữ liệu RTK để xác định vị trí thời gian thực trên thực địa;

Dữ liệu RINEX được sử dụng để xác định vị trí nhờ xử lý sau.

Trong trường hợp trạm CORS gắn kết với các trạm IGS, dữ liệu RINEX được sử dụng để phát triển các mạng lưới GNSS độ chính xác cao dựa trên các sản phẩm dịch vụ do tổ chức IGS cung cấp.

Như vậy mạng lưới các trạm CORS ở các quốc gia thường được chia thành hai phần: Một phần được sử dụng để hỗ trợ các tổ chức IGS/EUREF thực hiện các chức năng của mình, một phần được sử dụng cho các mục đích quốc gia.

Những sản phẩm được cung cấp bởi IGS cùng với mạng lưới các trạm CORS quốc gia đã tạo tiền đề quan trọng để phát triển phương xác định vị trí (định vị) điểm độ chính xác (Presice Point Positioning) nhằm tăng nhanh việc nhận được các kết quả độ chính xác cao trong ITRF (trong vòng 30 phút).

Phương pháp xác định vị trí (định vị) điểm độ chính xác (Presice Point Positioning) bản chất là phương pháp định vị điểm tuyệt đối được xác định từ các vệ tinh GNSS có các tọa độ không gian đã biết độ chính xác cao.

Dịch vụ cung cấp các tọa độ không gian và các số hiệu chỉnh đồng hồ vệ tinh độ chính xác cao của các vệ tinh GNSS là tiền đề để phát triển phương pháp định vị điểm với mục đích xác định vị trí của một trạm thu đơn độc với độ chính xác từ mức $\mathrm{dm}$ đến $\mathrm{cm}$ dựa trên các kết quả đo GNSS và các dịch vụ nêu trên.

Các dịch vụ nêu trên nhận được:

Từ Trung tâm xử lý các dữ liệu GNSS của các mạng lưới CORS hoặc NRTK-CORS;

Từ tổ chức IGS thông qua các sản phẩm bổ sung siêu nhanh (ultra - rapid products) bao gồm các tọa độ không gian với độ chính xác ở mức \pm $3 \mathrm{~cm}$ và các số hiệu chỉnh đồng hồ vệ tinh với độ chính xác ở mức \pm 0.2 ns của các vệ tinh GNSS sau khi thu các tín hiệu từ các vệ tinh GNSS trong vòng $3 \mathrm{~h}$ hoặc các sản phẩm bổ sung nhanh (rapid products) bao gồm các tọa độ không gian với độ chính xác ở mức $\pm 2.5 \mathrm{~cm}$ và các số hiệu chỉnh đồng hồ vệ tinh với độ chính xác ở mức \pm 0.1 ns của các vệ tinh GNSS sau khi thu các tín hiệu từ các vệ tinh GNSS trong vòng $7 \mathrm{~h}$.

Để đạt được độ chính xác ở mức cao hơn dm, ngoài việc sử dụng lịch vệ tinh chính xác, các số hiệu chỉnh đồng hồ vệ tinh, các số hiệu chỉnh tầng đối lưu khô, các trị đo giả cực ly và pha phải được hiệu chỉnh bởi độ lệch anten vệ tinh, hiệu ứng triều Trái đất cứng, sức tải đại dương, các tham số quay của Trái đất và hiệu ứng tâm sai tương đối và hiệu ứng Sagnac (El-Diasty, M., M. Elsobeiye, 2015). Trong thực tế áp dụng phương pháp PPP để xác định vị trí máy thu, chúng ta có thể đạt được độ chính xác ở mức cm khi sử dụng static mode và ở mức $\mathrm{dm}$ khi sử dụng linematic mode với thời gian đo khoảng 30 minutes (Bisnath, S., and Y. Gao, 2009). Hiện 
nay, ngoài các dịch vụ GNSS của tổ chức IGS, một trong những yếu tố phát triển phương pháp PPP là sự tồn tại đa vệ tinh GNSS (multiple GNSS) như GPS, GLONASS, GALEO, COMPASS. Đây là yếu tố đảm bảo có đủ số lượng vệ tinh để xử lý dữ liệu GNSS trong khi thời gian đo rất hạn chế chỉ trong vòng 30 phút. PPP (Precese Point Positioning) là kỹ thuật xác định vị trí độ chính xác cao khi chỉ sử dụng một máy thu dựa trên lịch vệ tinh chính xác và số cải chính đồng hồ vệ tinh chính xác đã biết trong thời gian thực (Farah,A., 2017). Kỹ thuật PPP được phát triển nhờ các dịch vụ cung cấp lịch vệ tinh chính xác và số cải chính đồng hồ vệ tinh chính xác bởi tổ chức IGS (Kouba, J., Héroux, P., 2001; IGS (2017)) và các tổ chức khác và sự xuất hiện các hệ thống đa vệ tinh trên thế giới. Với việc phát triển mạnh mẽ của việc áp dụng phương pháp PPP trong thời gian thực, tổ chức IGS đã cung cấp Dịch vụ thời gian thực (IGS Real Time Service - IGS-RTS). Nhiều phần mềm triển khai phương pháp PPP được phát triển, ví dụ CSRS-PPP của Cơ quan Tài nguyên Canada (Canadian Spatial Reference System Precise Point Positioning), GAPS (GPS Analysis and Positioning Software) của Rodrigo Leandro (University of New Brunswick), APPS (Automatic Precise Positioning Service) của Phòng Thí nghiệm phản lực (Jet Propulsion Laboratory - JPL) .V.V...

Khi áp dụng phương pháp PPP đối với các tín hiệu vệ tinh một tần số $\mathrm{L}_{1}$, ngoài lịch vệ tỉnh và số hiệu chỉnh đồng hồ vệ tinh độ chính xác cao chúng ta còn cần dịch vụ các số hiệu chỉnh tầng điện ly. The (Farah,A., 2017), CSRS - PPP (the Canadian Spatial Reference System - Precise Point Positioning) đảm bảo cung cấp các quỹ đạo vệ tinh chính xác và các cải chính đồng hồ vệ tinh chính xác đối với các GPS/GLONASS cho các trị đo giả cự ly và pha của các sóng mang $L_{1}$ và $L_{2}$ hoặc trị đo giả cự ly của sóng mang $L_{1}$ từ tổ chức IGS; các số cải chính tầng điện ly đối với sóng mang $\mathrm{L}_{1}$ được tích hợp từ bản đồ điện ly toàn cầu với khoảng thời gian $2 \mathrm{~h}$ trong format IONEX của tổ chức IGS với độ chính xác ở mức $\pm 2-8$ TECU (sai số khoảng $30 \mathrm{~cm}-1 \mathrm{~m}$ ); tổ hợp điện ly tuyến tính bậc 2 cho các trị đo hai tần số; các độ lệch tâm pha vệ tinh và máy thu trong IGS ANTEX; các số cải chính tầng đối lưu được tính theo mô hình Hopfield với hàm ánh xạ GMF (Global Mapping Function).

Khi áp dụng phương pháp PPP để xử lý các sóng mang hai tần số $\mathrm{L}_{1}$ và $\mathrm{L}_{2}$ người ta sử dụng rộng rãi sóng mang tự do điện ly $\mathrm{L}_{3}$ (Héroux, $\mathrm{P}$., J. Kouba, 2001; El-Diasty, M., M. Elsobeiye, 2015; Ocalan, T., 2016; Krzan, G., and P. Przestrzelsk, 2016). Khi đó chúng ta chủ yếu chỉ cần các dịch vụ tổ hợp điện ly tuyến tính bậc 2 cho các trị đo hai tần số; các độ lệch tâm pha vệ tinh và máy thu trong IGS ANTEX.

Trong bài báo khoa học này sẽ trình bày một số vấn đề cần đẩy mạnh nghiên cứu nhằm phát triển công nghệ GNSS ở nước ta khi đã có mạng lưới các trạm CORS.

\section{Giải quyết vấn đề}

\subsection{Các sản phẩm của tổ chức IGS và các tổ chức khác}

Khi xác định vị trí điểm bởi một máy thu, từ các dữ liệu GNSS thu được, theo (GPS-SPS (2008)), do các sai số trong lịch vệ tinh, đồng hồ vệ tinh, ảnh hưởng của tầng điện ly, tầng đối lưu, hiện tượng đa đường truyền và các nguồn sai số khác, độ chính xác của vị trí mặt bằng chỉ đảm bảo $\leq 13 \mathrm{~m}$ và độ chính xác của độ cao chỉ đảm bảo $\leq 22 \mathrm{~m}$.

Do đó để xác định vị trí điểm bởi một máy thu với độ chính xác cao ở mức từ $\mathrm{dm}$ đến $\mathrm{cm}$ phải sử dụng các dịch vụ GNSS do tổ chức IGS cung cấp.

Theo (Kouba, J., Héroux, P., 2001), lịch vệ tinh IGS cuối cùng (Final) được kết hợp từ các kết quả tính toán của 7 trung tâm phân tích IGS và có sẵn sau 11 ngày. Lịch vệ tinh IGS nhanh (Rapid) được kết hợp sau 17 h, còn lịch vệ tinh IGS Ultra-rapid có sẵn sau khi đo vài giờ. Độ chính xác của lịch vệ tinh và số cải chính đồng hồ vệ tinh do tổ chức IGS cung cấp được trình bày chi tiết trong (Hà Minh Hòa, Nguyễn Ngọc 
Lâu, 2013' Kouba, J., Héroux, P., 2001; IGS (2017)).

Ngoài các dịch vụ về lịch vệ tinh chính xác và các số hiệu chỉnh đồng hồ vệ tinh chính xác, tổ chức IGS còn cung cấp nhiều dịch khác. Chúng ta sẽ nghiên cứu các dịch vụ này.

\section{Các độ lệch tâm pha anten vệ tinh}

Các tọa độ vệ tinh chính xác và các số hiệu chỉnh đồng hồ vệ tinh chính xác của IGS được quy chiếu vào tâm vật chất của vệ tinh, trong khi đó các quỹ đạo vệ tinh trong Thông báo đạo hàng của vệ tinh lại quy chiếu vào tâm pha anten (Kouba, J., Héroux, P., 2001). Tuy nhiên các trị đo GNSS lại được tính từ tâm pha anten. Do đó để quy chiếu các trị đo GNSS vào tâm vật chất của vệ tinh, chúng ta cần phải biết các độ lệch của tâm pha vệ tinh so với tâm vật chất của vệ tinh (xem Hình 1).

Các tâm pha của tuyệt đại đa số vệ tinh là các độ lệch trong vật thể với trục $\mathrm{z}$ hướng xuống Trái đất, còn trục $X$ hướng về phía Mặt trời và nằm trong mặt phẳng chứa Mặt trời (Kouba, J., Héroux, P., 2001). Quy định IGS đối với các độ lệch (đơn vị mét) của tâm pha anten trong khung quy chiếu cố định của vệ tinh:

$\begin{array}{llccc} & & \mathrm{X} & \mathrm{Y} & \mathrm{Z} \\ \text { Block } & \text { II/IIA: } & 0,279 & 0,000 & 1,023 \\ \text { Block } & \text { IIR: } & 0,000 & 0,000 & 0,000\end{array}$

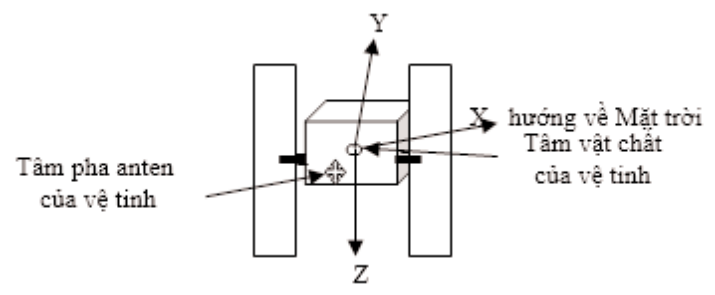

Hinh 1

Hiện nay, theo (Krzan, G., and P. Przestrzelsk, 2016), các độ lệch tâm pha anten vệ tinh không cần hiệu chỉnh, bởi vì các quỹ đạo và các số hiệu chỉnh đồng hồ vệ tinh được sử dụng đã quy chiếu về tâm pha anten vệ tinh.

\section{Hiệu chỉnh sự kích động pha}

Theo (Kouba, J., Héroux, P., 2001), vệ tinh phát sóng vô tuyến phân cực tròn bên phải (Right Circularly Polarized - RCP) và các trị đo pha phụ thuộc vào định hướng lẫn nhau giữa vệ tinh và máy thu. Sự quay của anten vệ tinh quanh trục của nó làm pha thay đổi đi một chu kỳ, Hiệu ứng này được gọi là sự kích động pha (phase wind - up) (Wu, J,T., S.C. Wu, G.A. Hajj, W.I. Bertiger, and S.M. Listen, 1993).

Nếu máy thu cố định, thì nó sẽ không quay và được định hướng quy chiếu (luôn là hướng Bắc). Tuy nhiên quan hệ hình học máy thu - vệ tinh thay đổi do vệ tinh chuyển động. Do vị trí Mặt trời thay đổi trên đường Hoàng đạo (eclipse), nên vệ tinh thường xuyên phải định hướng lại các panel pin mặt trời về phía Mặt trời. Điều này được gọi là "noon" (khi đường thẳng từ Mặt trời cắt vệ tinh trước, sau đó cắt tâm trái đất) và "midnight turns" (khi đường thẳng từ Mặt trời cắt tâm trái đất trước, sau đó cắt vệ tinh). Điều này được miêu tả là sự quay anten đi một góc nhỏ hơn 30'. Các dữ liệu đo pha phải hiệu chỉnh do hiệu ứng này (Bar-Sever, Y.E., 1996).

Theo (PPP standards), khi muốn đạt độ chính xác $\pm 4 \mathrm{~cm}$ với chiều dài baseline $4000 \mathrm{~km}$, số hiệu chỉnh kích động pha có ý nghĩa. Việc bỏ qua số hiệu chỉnh này có thể gây ra sai số trong việc xác định IGS quỹ đạo vệ tinh/đồng hồ vệ tinh ở mức dm. Kể từ năm 1994, các Trung tâm phân tích IGS đã sử dụng số hiệu chỉnh kích động pha để xác định IGS quỹ đạo vệ tinh/đồng hồ vệ tinh.

Trong tài liệu (Hà Minh Hòa, Nguyễn Ngọc Lâu, 2013) đã trình bày nhiều công thức hiệu chỉnh các hiện tượng địa động lực, ví dụ Triều trái đất cứng, Triều cực trái đất, Hiệu ứng sức tải áp suất khí quyển và Hiệu ứng sức tải đại dương. Bên cạnh việc tính đến các hiện tượng nêu trên, việc tính đến Ảnh hưởng của tầng đối lưu và Ảnh hưởng của tầng điện ly cũng được trình bày chi tiết trong tài liệu trên.

Như đã trình bày trong (Hà Minh Hòa, Nguyễn Ngọc Lâu, 2013), sự chậm thời gian (thời trễ) truyền tín hiệu vệ tinh trong tầng điện ly được gây ra do sự có mặt của các điện tử tự 
do. Sự chậm thời gian nêu trên tỷ lệ nghịch với bình phương của tần số sóng mang. Theo (Lachapelle G., Falkeyberg W., Newfelli D. and Kielland P.,1989) trong phương pháp đo GPS tương đối, hiệu các số cải chính do thời trễ ở tầng điện ly đối với các trị đo GPS từ hai điểm đầu của baseline phụ thuộc vào trạng thái của tầng điện ly trong khoảng thời gian thu tín hiệu vệ tinh và khoảng cách giữa hai điểm đó.

Một số kết quả nghiên cứu đã chỉ ra rằng chiều dài baseline $3 \mathrm{~km}$ ở vĩ độ trung bình là giới hạn thực tế của việc sử dụng máy thu một tần số và không cải chính do ảnh hưởng của tầng điện ly (Medvedev P.P., Baranov I. S., 1992).

Do sự biến thiên bất ngờ không dự đoán trước được của tầng điện ly, nên vào năm 1985 các nhà trắc địa đòi hỏi phải sử dụng máy thu hai tần số trong đo đạc độ chính xác cao. Tuy nhiên khi đó vẫn còn ảnh hưởng tầng điện ly bậc cao và sự ảnh hưởng này không bị triệt tiêu bởi việc sử dụng các dữ liệu đo GPS trên hai tần số. Trong các tổ hợp tuyến tính của các trị đo GPS hai tần số ảnh hưởng tầng điện ly quan hệ mạnh với các trị nguyên đa trị. Do đó các tổ hợp tuyến tính này không đại diện cho sự ảnh hưởng thuần tuý của tầng điện ly. Việc tính đến sự ảnh hưởng của tầng điện ly đến các kết quả đo GNSS được các nhà trắc địa quan tâm dặc biệt.

Chúng ta nghiên cứu sâu thêm về tầng điện ly. Như đã trình bày trong (Hà Minh Hòa, Nguyễn Ngọc Lâu, 2013), sóng mang không bị ảnh hưởng của tầng điện ly là sóng mang và để giải đa trị chúng ta kết hợp các trị đo và

Hiện nay phương trình sóng mang không bị ảnh hưởng của tầng điện ly là sóng mang được sử dụng rộng rãi trong phương pháp PPP. Việc kết hợp các trị đo và được sử dụng để giải đa trị trong quá trình xử lý các dữ liệu GNSS theo phương pháp PPP.

\section{Các files dữ liệu của IGS}

Format name IGS Product/Sampling

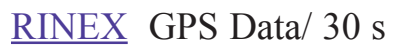

RINEX Clock Sat./Sta. Clock/5 min/30s

sp3 Orbits/Clocks/ 15 min.

IGS ERP Format IGS ERP/ 1 day

INEX Sta. Pos.(ERP) / 7(1) day

SINEX-tropo ext. Tropo. ZPD $2 \mathrm{~h} / 5 \mathrm{~min}$

IONEX Iono. maps/sat DCB $2 \mathrm{~h}$

\section{Phương pháp quy nguyên các trị nguyên đa trị}

Đối với các sóng mang hai tần số, việc giải đa trị (Ambiguity Resolution) được thực hiện nhờ các phương trình hiệu kép của các sóng mang $\mathrm{L}_{3}$ và $\mathrm{L}_{5}$ theo phương pháp bình phương nhỏ nhất (xem Hà Minh Hòa, Nguyễn ngọc Lâu, 2013). Trong trường hợp này, các trị nguyên đa trị được xác định là các số thực và có các giá trị rất gần với các số nguyên. Việc quy nguyên được thực hiện trực tiếp khai các giá trị tuyệt đối của số thực của trị nguyên đơn trị và sai số trung phương của nó nhỏ hơn 0.15 chu kỳ (Talbot N. C., 1991). Khi chúng ta không thực hiện được việc quy nguyên, lời giải trị nguyên đa trị là lời giải Float và cho các kết quả xác định các thành phần của các vectơ baseline với độ tin cậy thấp. Khi chúng ta thực hiện thành công việc quy nguyên, lời giải trị nguyên đa trị là lời giải Fixed và cho các kết quả xác định các thành phần của các vectơ baseline với độ tin cậy cao.

Tuy nhiên, trong trường hợp xử lý các dữ liệu GNSS một tần số trên các cạnh ngắn (không lớn hơn $20 \mathrm{~km}$ ), các trị nguyên đa trị được giải từ các phương trình hiệu kép của sóng mang $\mathrm{L}_{1}$ theo phương pháp bình phương nhỏ nhất, các trị nguyên đa trị được xác định là các số thực và có các giá trị chênh khá lớn so với các số nguyên tương ứng. Do đó, đối với việc quy nguyên các trị nguyên đa trị trong trường hợp xử lý các dữ liệu GNSS một tần số trên các cạnh ngắn trở thành vấn đề kỹ thuật phức tạp.

Chúng ta sẽ nghiên cứu một số phương pháp quy nguyên các trị nguyên đa trị trong trường hợp giải đa trị đối với các sóng mang một tần số 
$\left(\mathrm{L}_{1}\right)$ trên các khoảng cách ngắn.

\section{Phương pháp Tự quy nguyên (integer Bootstrapping)}

Đối với công nghệ GNSS, hệ phương trình số cải chính của các trị đo pha được biểu diễn dưới dạng (Teunissen, P., Joosten, P, and Tiberius, C., 2002):

$$
V+y=A . a+B . b+e
$$

ở đây y - vectơ các trị đo hiệu kép của các trị đo pha và có kích thước $\mathrm{m} ; \mathrm{V}$ - vectơ các số cải chính của các trị đo hiệu kép của các trị đo pha; $\mathrm{a}$ và $\mathrm{b}$ là các vectơ ẩn số cần tìm với các kích thước $\mathrm{n}$ và $\mathrm{p}$ một cách tương ứng; $\mathrm{e}$ - vectơ nhiễu.

Vectơ y luôn chứa các trị đo hiệu kép “được đo trừ đi được tính" một tần số (single), hai tần số (dual) hoặc tần số bội (triple - frequancy). Khi vectơ a là các trị nguyên đa trị hiệu kép, thì nó được biểu diễn trong đơn vị chu kỳ thay cho đơn vị mét. Vectơ a là các số nguyên và $\mathrm{a} \in \mathrm{Z}^{\mathrm{n}}$, ở đây $\mathrm{Z}$ là không gian nguyên $\mathrm{n}$ chiều. Vectơ $\mathrm{b}$ chứa các ẩn số còn lại như các hiệu kép của các cự ly hình học, các tham số độ trễ khí quyển (đối lưu, điện ly). Vectơ $b$ là các số thực và $b \in R^{p} \cdot Q_{y}$ là ma trận tương quan của vectơ $\mathrm{y}$.

Bài toán giải đa trị đối với hệ phương trình (94) được thực hiện qua 03 bước. Ở bước 1 , khi giải hệ (1) theo nguyên tắc bình phương nhỏ nhất tiêu chuẩn $V^{T} \cdot Q_{y}^{-1} \cdot V=$ min, chúng ta nhận được các vectơ nghiệm chứa các số thực "象 dưới dạng:

$$
\left[\begin{array}{l}
\hat{a} \\
\hat{b}
\end{array}\right]=\left[\begin{array}{cc}
Q_{\hat{a}} & Q_{\hat{a} \hat{b}} \\
Q_{\hat{b} \hat{a}} & Q_{\hat{b}}
\end{array}\right] \cdot\left[\begin{array}{c}
A^{T} \cdot Q_{y}^{-1} \\
B^{T} \cdot Q_{y}^{-1}
\end{array}\right] \cdot y
$$

và ma trận tương quan

ở đây

$$
\left[\begin{array}{cc}
Q_{\hat{a}} & Q_{\hat{a} \hat{b}} \\
Q_{\hat{b} \hat{a}} & Q_{\hat{b}}
\end{array}\right],
$$

$$
\left[\begin{array}{ll}
Q_{\hat{a}} & Q_{\hat{a} \hat{b}} \\
Q_{\hat{b} \hat{a}} & Q_{\hat{b}}
\end{array}\right]=\left[\begin{array}{ll}
A^{T} Q_{y}^{-1} \cdot A & A^{T} Q_{y}^{-1} \cdot B \\
B^{T} Q_{y}^{-1} \cdot A & B^{T} Q_{y}^{-1} \cdot B
\end{array}\right]^{-1} .
$$

Vectơ nghiệm $\hat{a}$ là vectơ của các số thực và được gọi là vectơ nghiệm "động - float" và không đạt được độ chính xác cao. Ở bước 2 , với mục đích nhận được nghiệm "xác định - fixed" với độ chính xác cao, chúng ta phải biến đổi để trị nguyên đa trị động $\hat{a}$ thành trị nguyên đa trị là giá trị nguyên $\breve{a}$.

Chúng ta giải quyết bài toán trên dưới điều kiện $\min (\hat{a}-\breve{a})^{T} \cdot Q_{\hat{a}}^{-1} \cdot(\hat{a}-\breve{a})$ với $a ̆ \in Z^{n}$. Với mục đích quy nguyên vectơ $\hat{a}$ khi tôn tại phép ánh xạ M: $\mathrm{R}^{\mathrm{n}} \longrightarrow \mathrm{Z}^{\mathrm{n}}$ để chuyển không gian thực $\mathrm{n}$ chiều $\mathrm{R}^{\mathrm{n}}$ sang không gian nguyên $\mathrm{Z}^{\mathrm{n}}$ chúng ta có:

$$
\breve{a}=M[\hat{a}], \quad \breve{a} \in Z^{n}, \hat{a} \in R^{n} .
$$

Do các thành phần của vectơ $\hat{a}$ phụ thuộc nhau rất chặt, nên quá trình làm tròn cần phải tính đến sự phụ thuộc nêu trên. Trong tài liệu (Teunissen $\mathrm{P}, 1998$ ) đã đề xuất quá trình tự quy nguyên như sau:

$$
\begin{aligned}
& \breve{a}_{B, 1}=\left[\hat{a}_{1}\right\rfloor, \\
& \breve{a}_{B, 2}=\left[\hat{a}_{2 \mid 1}\right]=\left[\hat{a}_{2}-\sigma_{\hat{a}_{1} \hat{a}_{2}} \cdot \sigma_{\hat{a}_{1}}^{2} \cdot\left(\hat{a}_{1}-\breve{a}_{B, 1}\right)\right], \\
& \breve{a}_{B, n}=\left[\hat{a}_{n \mid n-1}\right]=\left[\hat{a}_{n}-\sum_{i=1}^{n-1} \sigma_{\hat{a}_{n}, \hat{a}_{i \mid I}} \cdot \sigma_{\hat{a}_{i \mid I}^{2}}^{2} \cdot\left(\hat{a}_{i \mid I}-\breve{a}_{B, i}\right)\right],
\end{aligned}
$$

ở đây ký hiệu [.] có nghĩa là làm tròn đến số nguyên gần nhất; $\hat{3}_{\hat{Z}}$ là trị nguyên đa trị thứ i được xác định từ các trị nguyên đa trị được làm tròn ở các bước trước, còn $I=\{1, \ldots, n-1\}$; chỉ số $\mathrm{B}$ là viết tắt của chữ Bootstrapping. Điều kiện làm tròn là $\left|\hat{a}_{i \mid I}-\breve{a}_{B, i}\right| \leq \frac{1}{2}$.

Quy trình tự quy nguyên nêu trên được sử dụng trong phương pháp TCAR (Three - Carrier Ambiguity Resolution) được đề xuất để xử lý các dữ liệu GALILEO (Forsell B., Martin Neira M., and Harris R., 1997; Vollath U., Birmbach S., and Landau H., 1998) và phương pháp CIR (Cascading Integer Resoluion) đối với các dữ liệu GPS (Hatch R., Jung J., Enge P., and Pervan B., 2000; Jung J., Enge P., and Pervan B., 2000).

Ngoài ra, nhiều phương pháp khác được sử 
dụng rộng rãi, ví dụ Phương pháp bình phương nhỏ nhất nguyên (Integet Least - Squares Method); phương pháp LAMBDA (Least-squares AMBiguity Decorrelation Adjustment - Bình sai làm giảm sự tương quan của các trị nguyên đa trị theo phương pháp bình phương nhỏ nhât).

\section{Kết luận}

Để triển khai phương pháp PPP, chúng ta cần chuẩn bị khá nhiều kiến thức mới về công nghệ GNSS. Do đó để phát triển công nghệ GNSS trong thời gian tới, việc nghiên cứu và trang bị nhiều kiến thức mới là điều rất cần thiết nhằm ứng dụng mạnh mẽ công nghệ GNSS trên cơ sở khai thác hiệu quả mạng lưới các trạm CORS hiện có. $\bigcirc$

\section{Tài liệu tham khảo}

[1]. Alber A.R., Rocken C. and Solheim F., 1997. GPS surveying with $1 \mathrm{~mm}$ precision using corrections for atmospheris slant path delay. Geophysical Research Letters, 24, 1850-1862, 1997.

[2]. Bar-Sever, Y.E., 1996. A new module for GPS yaw attitude control. Proceedings of IGS Workshop - Special Topics and New Directions, eds. G. Gendt and G. Dick, GeoforschunsZentrum, Potsdam, pp. 128-140.

[3].Bevis, M., Businger, S., Herring, T.A., Rocken, C., Anthes, A. , Ware, R.H., 1992. GPS meteorology: Remote Sensing of atmospheric water vapor using the Global Positioning System. Journal of geophysical Research, 97(D14), 15787 - 15801.

[4]. Beutler, G., Bock, H., Brockmanu, E., Dach, P., Frides, P., Gurtner, W., Hugentobler, U., Ineichen, D., Johnson, J., etc., 2001. Bernese GPS software version 4.2, Astronomical institute, University of Berne, January-2001.

[5]. Bisnath, S., and Y. Gao, 2009. Precise Point Positioning - A Poweful Technique With a Promising Future. GPS World, 51 p., 2009 April.

[6]. Blewitt, G., 1994. Atmospheric Loading Effects and GPS Time-averaged Vertical
Positions AGU 1994 Spring Meeting, EOS, Transaction, American Geophysical Union, Vol. 75, No 16 Supplement, PP. 104 - 105.

[7]. Brunner, F. K. , Trogoning, P., 1994. Tropospheric propagation Effects in GPS height results using meteological observation. Aust. J.Geod.photogam.Serv, No 60, June, pp.49-65.

[8]. Chang, C. C., 1999. Geophysical effects on site diplacements for parmanent GPS tracking stations in Taiwan, Geomatics Research, Australia, No 71, Dec., PP. 1-18.

[9]. Chardlow, P.J., 1994. Propagation effects on precise GPS heighting. PH.D. thesis. Univercity of Notingham. Institute of engeineering surveying and space geodesy. 221p. 1994.

[10]. Chen, J. I., C.R. Wilson, R.J. Eanes and R.S. Nerem, 1999. Geophysical interpretation of observed geocenter variations. Jour. of geophysical Research, Vol. 104, No B2, PP. 2683 - 2690, February.

[11]. Chen, G., Herring, T.A., 1997. Effects of atmospheric azimuthal asymmetry on the analysis of space geodetic data. Journal of geophysical Research, 102(B9), 20489 - 20502.

[12]. Collins, P., Langley, R., 1999. Tropospheric delay: prediction for the WAAS user, GPS world, 52-58, 1999.

[13]. Davis, J.L., Elgered, G., Niell, A.E., Kuchn, C.E., 1993. Ground - based measurement of gradients in the "wet " radio refractivity of air. Radio Science. 28(6), 1003 - 1018.

[14]. Davis, J.L., 1986. Atmospheric propagation effects on radio interferometry. AFGL TR - 86 - 0243, Air Force geophysic Laboratory.

[15]. De Jonge, P.J., and Tiberius, C.C.J.M., 1996. The LAMBDA method for integer ambiguity estimation: implementation aspects. Publications of the Delf Geodetic Computating Centre, Technical trport LGR Series, No12, August 1996. Delf University of Technology, The Netherlands. 
[16]. Duan, J., Bevis, M., Chiswell, S., Businger, S., Rocken, C., Solhem, F., Hove, T.V., Ware, R.H., McClusky, S., Herring, T.A., King, R., 1996. GPS meteorology: Direct estimation of the absolute value of precipitable water. Journal of applied meteorology, 35, $830-838$.

[17]. El-Diasty, M., M. Elsobeiye, 2015. Precise Point Positioning Technique with IGS Real - Time Service (RTS) for Maritime Applications. Positioning, 6, 71-80. http://www.scrip.org/journal/pos. http://dx. doi.org/10.4236/pos.2015.64008

[18]. Farah, A., 2017. Variation of static PPP positioning accuracy using GPS-single frequency observations (Aswan, Egypt). Artificial Satellites, Vol. 52, No. 2, pp.19-26, doi: 10.1515/arsa-3017-0003.

[19]. Forsell, B., Martin - Neira, M., and Harris, R., 1997. Carrier phase ambiguity resolution in GNSS-2. In Proceedings ION GPS-97, pp. 1727 - 1736.

[20]. Gipson, J. M. and Ma, C., 1998. Site Diplacement due to variation in Earth Rotation. Jour. of Geophysical Research, Vol. 103, No. B4, PP. 7337 - 7350, 1998.

[21]. GPS-SPS (2008). GPS Standard Positioning Service (SPS) specifications. http:/www.gps.gov/technical/ps/2008-SPS-performance-standard.pdf

[22]. Gurtner, W., Beutler, G., Botton, S.,,Rothacher, M., Geiger, A.,Kable, H.G., Schneider, D., Wiget, A., 1989. The use of the Global Positioning System in Mountainous Areas, Manuscripta Geodaetica, 14 : 53-60

[23]. Hà Minh Hòa, Nguyễn Ngọc Lâu, 2013. Nguyên lý lý thuyết và thực tiễn của Trắc địa vũ trụ. Nhà Xuất bản Khoa học và Kỹ thuật, 276 trg., Hà Nội - 2013.

[24]. Hà Minh Hòa, 2018. Một số vấn đề hiện đại của Trắc địa vật lý, 964 trg. (Sách chuyên khảo). ISBN: 978- 604-67-1116-2, Nhà xuất bản Khoa học và Kỹ thuật.
[25]. Hatch, R., Jung, J., Enge, P., and Pervan, B., 2000. Civilian GPS: The benefit of three frequencies, GPS Solution, 3 (4), 1-9.

[26]. Héroux, P., J. Kouba, 2001. GPS Precise Point Positioning Using IGS Orbit Products. Phys, Chem. Earth (a), Vol.26, No. 6-8, pp. 573578.

[27]. Hofmann - Wellenhof, B., Lichtenegger, H., and Collins, J., 1994. Global Positioning System. Theory and Practice. Third, revised edition. Springer - Verlay - New York. April - 1994.

[28]. IGS (2017). International GNSS Service (IGS) products. http://igscb.jpl.nasa.gov/components/prods.html

[29]. Ihde, J., C. Bruyninx, 2008. Developments of the EUREF GNSS Services and Reference Networks. ICG-03 Meeting, 8-12 December 2008, Pasadena, USA.

[30]. Jung, J., Enge, P., and Pervan, B., 2000. Optimization of cascade integer resolution with three civil GPS frequencies. In Proceedings of ION - GPS 2000, Salt Lake City, UT, USA, pp. $2191-2200$.

[31]. King, R. W., Master, E.G., Rizos, C., Stolz, A., Collins, J., 1985. Surveying with Global Positioning System - GPS, The university of New South Wales, Australia.

[32]. Kouba, J., Héroux, P., 2001. Precise point positioning using IGS orbit and clock products. GPS Solutions. 5(2): 12-28.

[33]. Krzan, G., and P. Przestrzelsk, 2016. GPS/GLONASS Precise Point Positioning With IGS Real - Time Service Products. Acta Geodyn. Geomater., Vol. 13, No. 1 (181): 69-81. doi: 10.13168/AGG.2015.0047.

[34]. Lachapelle, G., Falkeyberg, W., Newfelli, D. and Kielland, P., 1989. Marine DGPS using code and carrier in a multipath environment, Proceedings of the second International Technical Meeting of the Satellite Division of Institute of Navigation, P. 343-347. 
Colorado Springs. Colorado, USA, September.

[35]. MacMillan, D.S., 1995. Atmospheric gradients from Very Long Baseline Interferometry observations. Geophysical Research Letters, . 22, 1041 - 1044.

[36]. MacMillan, D.S., Ma, C., 1997. Atmospheric gradients and VLBI Terrestrial and Celestial Reference frame. Geophysical Research Letters , 24(4) , 453 - 456.

[37]. Mecarthy, D. D., 1996. IERS coventions. International Earth Rotation Services. Technical Note 21, Obervatoire de Paris. 1996.

[38]. Mendes, V.B. and Langley, R.B., 1994. "A Comprehensive analysis of mapping function used in modeling tropospheric propagation delay in space geodetic data", International Symposium on Kinematic systems in Geodesy, Geomatics and Navigation, Canada, August 30September 2, 1994.

[39].Monico, J. F. G., V. Ashkenazi, and T. Moore, 1997.“High Precision GPS Network with Precise Ephemerides and Earth Body Tide Model”. Revista Brasileira de Geofisica, 15(2), 155-160.

[40]. Niell, A.E., 1996.“Global mapping functions for the atmospheric delay at radio wavelengths", Journal of geophysical research, vol 101, No B2, pages 3227-3246.

[41]. Ocalan, T., 2016. Accuracy Assessment of GPS Precise Point Positioning (PPP) Technique Using Defferent Web-based Online Service In a Forest Environment. Prethodno priopcenje - Preliminary communication. Smarski list, 7-8: 357-368.

[42].

PPP standards. www.navipedia.net/indes.php/PPP_standards

[43]. Rodrigo F. Leandro, Langley, R.B. and Santos, M.C., 2007. "UNB3m_pack: a neutral atmosphere delay package for radiometric space technique", GPS solution.

[44]. Saastamoinen, J., 1972. Atmospheric correction for the troposphere and stratosphere in radio ranging of satellites. In : Use of artificial satellites for Geodesy. Geophysical Monograph , 15 , Americal Geophysical Union, Wasington D.C.

[45]. Satirapod, C., J. Wang, and C. Rizos, 2003. Comparing different GPS data processing techniques for modeling residual systematic errors. J. Surv. Eng., 129 (4), 129-135.

[46]. Tralli, D.M., Lichten, S.M., 1990. Stochastic estimation of tropospheric path delays in Global Positioning System geodetic measurement. Bulletin geodesique, 64,127 159. 1990.

[47]. Talbot, N. C., 1991. "Sequential Phase Ambiguity Resolution for Real Time Static Differential GPS Positioning", Manuscripta Geodaetica, 16, 274- 282.

[48]. Teunissen, P., 1993. Least-squares estimation of the integer GPS ambiguities. In $I A G$ General Meeting, Invited Lecture, Section IV Theory and Methodology, Beijing, China.

[49]. Teunissen, P., 1995a. The invertible GPS ambiguity transformations. Manuscripta Geodeticae, 20, 489-497.

[50]. Teunissen, P., 1995b. The Least squares ambiguity decorrelation adjustment: a method for fast GPS integer ambiguity estimation. Journal of Geodesy, 70, 65 - 82.

[51]. Teunissen, P., 1998. Success probability of integer GPS ambiguity rounding and bootstrapping. Journal of Geodesy, 72: 606 - 612.

[52]. Teunissen, P., 2001. GNSS ambiguity bootstrapping: Theory and application. In Proceedings of: International Symposium on Kinematic Systems in Geodesy; Geomatics and Navigation, Banff, Canada, pp. 246-254.

[53]. Teunissen, P., Joosten, P, and Tiberius, C., 2002. A comparision of TCAR, CIR and LAMBDA GNSS ambiguity resolution. ION GPS 2002, 24-27, September, Portland, OR, pp. 2799-2808

(Xem tiếp trang 50) 